\title{
Automatic Detection of Luminal Borders in IVUS Images by Magnitude-Phase Histograms of Complex Brushlet Coefficients
}

\author{
Amin Katouzian, Student Member, IEEE, Elsa D. Angelini, Member, IEEE, Bernhard Sturm, and \\ Andrew F. Laine, Fellow, IEEE
}

\begin{abstract}
We present a new technique to delineate lumen borders in intravascular ultrasound (IVUS) volumes of images acquired with a high-frequency Volcano (Rancho Cordova, CA) $45 \mathrm{MHz}$ transducer. Our technique relies on projection of IVUS sub-volumes onto orthogonal directional brushlet functions. Through selective projection of IVUS sub-volumes images and their Fourier transforms, tissue-specific backscattered magnitudes and phases identified within brushlet coefficients. We take advantage of such characteristics and construct 2.5-dimensional (2.5-D) magnitudes-phase histograms of coefficients in the transformed complex brushlet domain that contain distinct peaks corresponding to blood and non-blood regions. We exploit these peaks to mask out coefficients that represent blood regions and ultimately detect the luminal border after spatial regularization employing a parametric deformable model. We quantify our results by comparing them to manually traced borders by an expert on 2 datasets, containing 108 frames. We show that our approach is well suited for isolating coherent (i.e. plaque) structures from incoherent (i.e. blood) ones in IVUS pullbacks and detecting the lumen border, a challenging problem particularly in images acquired with high frequency transducers.
\end{abstract}

\section{INTRODUCTION}

$\mathrm{O}$ FTEN, patients with chest pain and high cardiac risk factors undergo percutaneous coronary intervention (PCI) procedures. As a result, a catheter is inserted from the femoral artery toward potential sites of coronary occlusions to open up blocked artery with a stent or inflating a balloon. An interventional cardiologist may also deploy an IVUS catheter to acquire cross sectional images of arterial walls and atherosclerotic plaque structures. During such a procedure, hundreds to thousands of IVUS images are recorded. Therefore automatic detection of arterial wall and luminal borders has become a subject of study for the past decade. We can categorize each developed algorithm based on its application, domain of analysis, transducer center frequency, dimensionality, and employed technique as listed in Table I.

Manuscript received April 23, 2010. This work was supported by Volcano Corporation (Rancho Cordova, CA).

A. Katouzian is with the Heffner Biomedical Imaging Lab in the Department of Biomedical Engineering of Columbia University, New York, NY USA, (phone: 212-854-5996, Fax: 212-854-5995, email: ak2432@,columbia.edu).

E. D. Angelini is an Associate Professor of Computer Science Department at Telecom ParisTech, Paris, France,

(email: elsa.angelini@telecom-paristech.fr).

B. Sturm is Senior Research Scientist at Volcano Corporation, Rancho Cordova, CA USA, (email: bsturm@volcanocorp.com).

A. F. Laine is Director of Heffner Biomedical Imaging Lab, Professor and Vice Chair of Department of Biomedical Engineering at Columbia University, New York, NY USA, (email: Laine@,columbia.edu).
Generally speaking, detection of vessel wall borders is less difficult than lumen borders since the vessel media consists of smooth muscle cells and does not reflect IVUS signals. It therefore appears as a dark region on IVUS images, which can be used as a marker to detect the vessel wall. In contrast, due to high scattering from red blood cells inside the lumen, detection of a luminal border is a more formidable challenge especially when a high-frequency transducer is used. Our group previously presented a multiscale brushlet based technique to filter IVUS images and characterize blood speckle patterns as a preprocessing step for lumen border detection [10]. We demonstrated that brushlet coefficients provided information about blood and non-blood regions and could be used to isolate static (i.e. plaque) patterns from dynamic (i.e. blood) ones over whole pullback acquisitions. We later used brushlet coefficients along with two geometrical features to classify blood regions in a supervised fashion [11]. In this paper, we show that tissue-specific backscattered magnitudes and phase information can be classified using brushlet coefficients, due to indirect projection of 3D IVUS sub-volumes through their Fourier transforms. We then use this property to establish a framework that is able to delineate luminal borders automatically without any use of thresholding parameters.

Table I. List of existing algorithms and their specifications. (VW: Vessel Wall, L: Lumen)

\begin{tabular}{|c|c|c|c|c|}
\hline $\begin{array}{l}\text { Authors } \\
\text { [Ref. \#] }\end{array}$ & $\begin{array}{c}\text { Implementation } \\
\text { Technique }\end{array}$ & Application & $\begin{array}{l}\text { Domain of } \\
\text { Analysis }\end{array}$ & $\begin{array}{c}\text { Tcx. Center } \\
\text { Frequency } \\
\text { (MHz) } \\
\end{array}$ \\
\hline $\begin{array}{c}\text { Sonka et al } \\
{[1]}\end{array}$ & 2D graph search & VW \&L & Cartesian & 30 \\
\hline $\begin{array}{l}\text { Shekhar et } \\
\text { al [2] }\end{array}$ & $\begin{array}{l}\text { 3D deformable } \\
\text { model }\end{array}$ & VW \&L & Cartesian & 30 \\
\hline $\begin{array}{c}\text { Plissiti et } \\
\text { al [3] }\end{array}$ & $\begin{array}{c}\text { 2D deformable } \\
\text { model }\end{array}$ & VW \&L & Cartesian & 30 \\
\hline $\begin{array}{l}\text { Cardinal et } \\
\text { al [4] }\end{array}$ & $\begin{array}{l}\text { 3D PDF-based } \\
\text { fast marching }\end{array}$ & VW \&L & Polar & 20 \\
\hline $\begin{array}{c}\text { Unal et al } \\
{[5]}\end{array}$ & $\begin{array}{c}\text { 2D/3D statistical } \\
\text { shape model }\end{array}$ & VW \&L & Polar & 20 \\
\hline $\begin{array}{l}\text { Hibi et al } \\
{[6]}\end{array}$ & $\begin{array}{c}\text { 3D } \\
\text { spatio/temporal } \\
\text { analysis } \\
\end{array}$ & $\begin{array}{l}\text { Blood noise } \\
\text { reduction }\end{array}$ & Polar & 40 \\
\hline $\begin{array}{l}\text { Ballocco et } \\
\quad \text { al [7] }\end{array}$ & $\begin{array}{c}\mathrm{D} \\
\text { spatio/temporal } \\
\text { analysis } \\
\end{array}$ & $\begin{array}{l}\text { Blood noise } \\
\text { reduction }\end{array}$ & Cartesian & 30 \\
\hline $\begin{array}{l}\text { Rotger et } \\
\text { al [8] }\end{array}$ & $\begin{array}{c}\text { 3D, Adaboost } \\
\text { classifier }\end{array}$ & $\begin{array}{c}\text { Blood } \\
\text { detection }\end{array}$ & $\begin{array}{c}\text { Longitudinal } \\
\text { Cut }\end{array}$ & Unknown \\
\hline $\begin{array}{l}\text { O'Malley } \\
\text { et al [9] }\end{array}$ & $\begin{array}{l}\text { 3D support vector } \\
\text { machine (SVM) }\end{array}$ & $\begin{array}{c}\text { Blood } \\
\text { detection }\end{array}$ & Polar & 40 \\
\hline
\end{tabular}

\section{DATA COLLECTION}

We collected IVUS grayscale images from two patients using a single element mechanically rotating $45 \mathrm{MHz}$ Revolution $^{\mathrm{TM}}$ transducer and an $\mathrm{s}^{\mathrm{TM}}$ imaging system manufactured by VOLCANO (Rancho Cordova, CA). The 


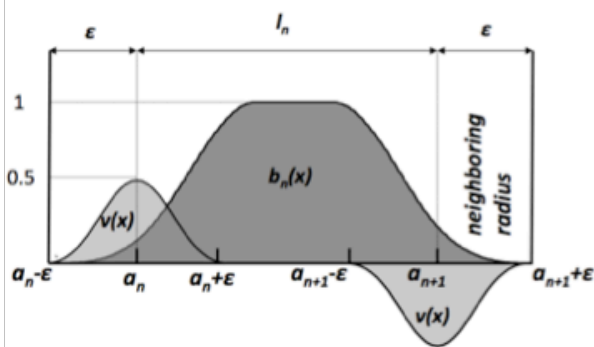

(a)

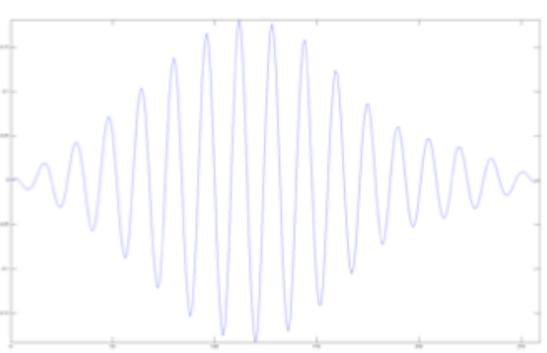

(b)

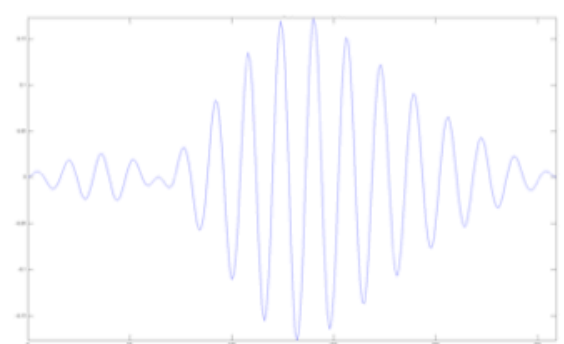

(c)

Fig. 1. Windowing functions $b(x)$ and $v(x)$ (a). The $\varepsilon$ parameter controls the localization degree of brushlet function in time and frequency domains. Real part (b) and imaginary part (c) of analysis $u_{n, j}$ brushlet function with $l_{n}=32, \varepsilon=16, j=8$.

catheter was inserted on top of a guide wire from the femoral artery toward the potential site of coronary arterial occlusion (i.e. right coronary artery (RCA), left anterior descending (LAD), left circumflex (LCX)) via the aorta. During image acquisition, the catheter is pulled back from distal to proximal locations with a speed of $0.5 \mathrm{~mm} / \mathrm{sec}$, acquiring 30 frames/second. Ultimately, each grayscale IVUS frame was constructed consisting of 500 radial lines that span $360^{\circ}$ and 500 samples/line after decimation and interpolation in radial and lateral directions, respectively. The original images, acquired in polar coordinates, were mapped to Cartesian coordinates to construct typical IVUS image matrices.

\section{Methodology}

We designed our approach based on what typically interventional cardiologists do to visually delineate the lumen border. They usually go back and forth among consecutive frames to be able to visually locate the lumen contour on a single frame. By doing so, blood speckle and plaque embody visually incoherent and coherent spatial patterns, respectively, suggesting a 3D processing approach. 3D brushlet analysis has been successfully employed to segregate static and dynamic textured structures within volumes of images in different applications [10-12].

\section{A. Brushlet Analysis}

We are particularly interested in orthogonal localized exponentials basis functions since they enable us to characterize valuable information about the direction of textures at different scales, frequencies, and locations. For this reason, authors in [13] introduced Brushlet basis by dividing the real axis into subintervals $\left[a_{n}, a_{n+1}\right]$ of length $l_{n}$ and constructing the complex orthonormal basis function $u_{n, j}(x)$ using two localized windowed functions $b_{n}(x)$ and $v(x)$ as follows:

$$
\begin{array}{r}
u_{n, j}(x)=b_{n}\left(x-l_{n} / 2\right) e_{n, j}(x)+v\left(x-a_{n}\right) e_{n, j}\left(2 a_{n}-x\right) \\
-v\left(x-a_{n+1}\right) e_{n, j}\left(2 a_{n+1}-x\right)
\end{array}
$$

where $e_{n, j}(x)=1 / \sqrt{l_{n}} e^{-2 i \pi j \frac{\left(x-a_{n}\right)}{l_{n}}}$. Figure 1 illustrates the windowing functions as well as the imaginary and real parts of $u_{n, j}(x)$. Given any one-dimensional signal $f$ in $L^{2}(R)$, its Fourier transform $\hat{f}$ can be projected onto the brushlet basis as $\hat{f}=\sum_{n} \sum_{j} \lambda_{n, j} u_{n, j}$ (2), where $\lambda_{n, j}$ are the brushlet coefficients. It has been shown that the projection of $\hat{f}$ onto the brushlet basis can be implemented in an efficient fashion using a folding technique and fast Fourier transform (FFT) [14].

\section{B. IVUS Signal Modeling In Fourier Domain and Assumptions}

Consider the measurements of an IVUS transducer during pullback. In this case, each acquisition line, $f$, contains information regarding tissues and flowing blood. We can distinguish the differences in the temporal patterns associated with each tissue and blood by differentiation between their distinct responses to IVUS signal. Hence, we take in the relative magnitudes and phases into the definition of the sources (i.e. blood and non-blood) and represent them in the transformed domain as:

$\hat{f}_{n, j}=\sum_{n} \sum_{j} \alpha_{n, j} e^{-i \omega \varphi_{n, j}} \hat{s}$

where $\varphi_{n, j}$ and $\alpha_{n, j}$ are the resulting phase and amplitude of tissue response $\hat{s}$ to ultrasound signal. Using Eq. 3 we rewrite Eq. 2 and obtain:

$\sum_{n} \sum_{j} \alpha_{n, j} e^{-i \omega \omega_{n}, \hat{s}}=\sum_{n} \sum_{j} \lambda_{n, j} u_{n, j}$

We call two functions $s_{1}$ and $s_{2}$ disjoint orthogonal if the frequency supports of their Fourier transforms, $\hat{s}_{1}$ and $\hat{s}_{2}$, are disjoint. In other words the point-wise product: $\hat{s}_{p} . \hat{s}_{q}=0, \forall p \neq q, \forall N$. Our working assumption is that this is a legitimate assumption in our IVUS application because 


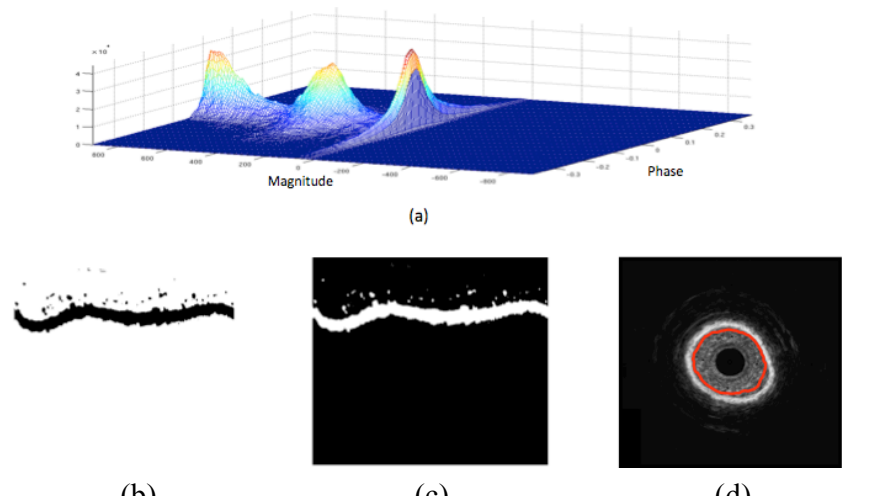

(b)

(c)

(d)

Fig. 2. Constructed magnitude-phase histogram (a), output of $\mathrm{K}$-means classifier for two classes $(\mathrm{b}, \mathrm{c})$, automated detected phantom wall (c).

each frequency bin is a function of only and only one tissue's response, which in our case would be blood or nonblood. Since brushlet basis are orthonormal, we can reorganize Eq. 4 in the following form:

$$
\lambda_{n, j}=\sum_{n} \sum_{j}\left\langle\alpha_{n, j} e^{-i \omega \varphi_{n, j}} \hat{s}_{n, j}, u_{n, j}\right\rangle
$$

Looking at Eq. 5, we realize that the phase of brushlet functions results in orientation selectivity in transformed space when separable tensor product is used while image phases and amplitudes associated with tissue responses are proportionally preserved in brushlet coefficients. We further hypothesize that the magnitude and phase of brushlet coefficients provide informative features for coherent (nonblood) and incoherent (blood) patterns so we can estimate the lumen border in transformed domain via selection of specific clusters of phases-magnitudes combinations avoiding any thresholding and reconstruction. In the rest of this section we present a classification framework to associate frequency-based clusters of brushlet coefficients with each tissue type (blood or non-blood).

\section{Construction of 2.5-D Magnitude-Phase Histogram in Complex Brushlet Space}

Once the IVUS sub-volumes of size $(X, Y, Z)$ are projected onto brushlet basis, we end up with sub-volumes of brushlet coefficients in the transformed domain. Corresponding to lower frequencies, we showed that the coefficients in the innermost cubes contain the most informative features regarding blood and non-blood textures. They can be summed up to span $360^{\circ}$ of spatially oriented information and construct the complete arterial wall and plaque in transformed domain $[10,11]$. We follow the same procedure and each IVUS frame in brushlet space, $\hat{F}$, contains all spatial coefficients. Taking the union of summed coefficients $\hat{F}=\bigcup_{k=1}^{X \times Y} \alpha_{k} e^{-i \varphi_{k}}$, we can write:

$\hat{F}(r, t)=\alpha(r, t) e^{-i \varphi(r, t)}, r=1, \ldots, X, t=1, \ldots, Y$
The magnitude and phase associated with each coefficient can be written as:

$$
(\alpha(r, t), \varphi(r, t))=(|\hat{F}(r, t)|, \measuredangle \hat{F}(r, t))
$$

A 2.5-D histogram can be constructed for every pair of $(\alpha(r, t), \varphi(r, t))$ in $(\alpha, \varphi)$ domain as follows. First, we define a mask for $(\alpha, \varphi)$ :

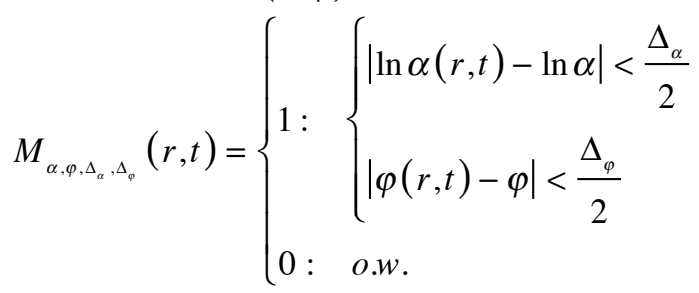

We are taking the difference between computed magnitude and phase of brushlet coefficients, $\alpha(r, t)$ and $\varphi(r, t)$ within $\frac{\Delta_{\alpha}}{2}$ of $\alpha$ and $\frac{\Delta_{\varphi}}{2}$ of $\varphi$ in the histogram, respectively, where $\Delta_{\alpha}$ and $\Delta_{\varphi}$ are the magnitude and phase resolution widths of the histogram. Then, the histogram can be defined as follows:

$$
h(\alpha, \varphi)=\sum_{r, t}\left|M_{\alpha, \varphi, \Delta_{\alpha}, \Delta_{\varphi}}(r, t) \hat{F}(r, t)\right|
$$

Our main interest is the locations of the histogram peaks and the surrounding region. As these shall be used to generate binary masks to label blood and non-blood regions.

\section{Segmentation of Coefficients and Recovery of Regional Peak Correspondences}

In order to localize the histogram peaks, we used a K-mean classifier to cluster the data in (9) $\left(\alpha_{\text {peak }}, \varphi_{\text {peak }}\right)$. The partitioning algorithm minimizes the sum of point-tocentroid distances, summed over all clusters. We assigned each point to the nearest cluster centroid and updated all centroids iteratively. Once the magnitudes and phases corresponding to the $Q$ principal peaks were found, we computed, $Q^{*}$, masks based the following $L^{2}$ norm in the histogram space,

$$
\begin{aligned}
& \forall\left\{\left(\alpha_{\text {peak }}^{l}, \varphi_{\text {peak }}^{l}\right), l=1, \ldots, K\right\} \\
& Q_{\alpha_{\text {peak }}^{l^{*},}, \varphi_{\text {peak }}^{l^{*}}}(r, t)=\left\{\begin{array}{lc}
1 ; & \min _{l}\left(\alpha(r, t)-\alpha_{\text {peak }}^{l}\right)^{2}+\left(\varphi(r, t)-\varphi_{\text {peak }}^{l}\right)^{2} \\
0 ; & \text { o.w. }
\end{array}\right.
\end{aligned}
$$

where (10) is minimum over all and is the number of tissues (classes). Note that the number of classes defined for the K-means classifier may not be necessarily the same as the number of peaks that we observe in the histogram. We expected that the magnitudes and phases of brushlet coefficients would provide some information about tissue types. Hence, $\left(\alpha_{\text {peak }}, \varphi_{\text {peak }}\right)$ corresponds to an approximate magnitude-phase for a tissue (class). In Eq. (10), we tried to identify specific $\left(\alpha_{\text {peak }}, \varphi_{\text {peak }}\right)$ among all magnitudes and 


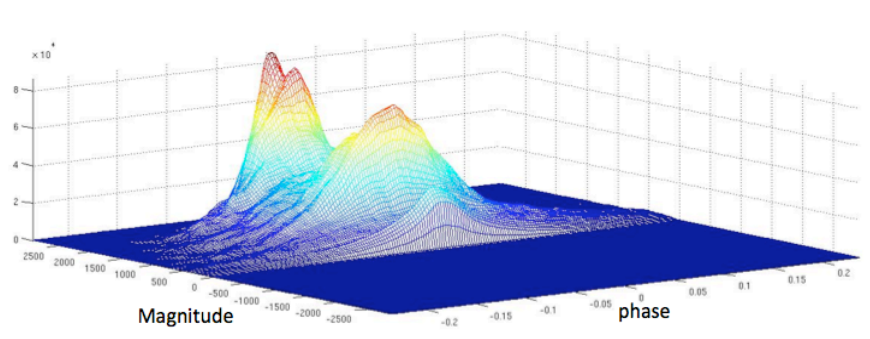

(a)

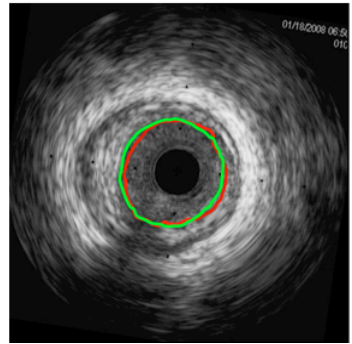

(b)

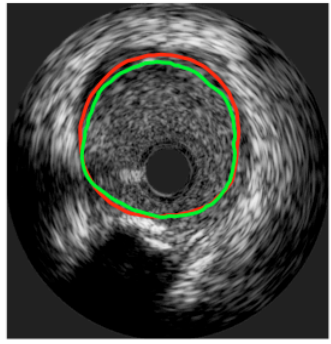

(c)

Fig. 3. Constructed magnitude-phase histogram for a single IVUS frame acquired in vivo (a), automated (red) and manual (green) traced borders imposed on two original grayscale IVUS images (b,c).

phases derived from the brushlet coefficients, $(\alpha, \varphi)$, and estimated the corresponding regions by masking the coefficients that exhibited the closest magnitude and phase to the approximated one. Once the desirable mask corresponding to blood regions was found (i.e., the one that contains zeros around the surface of the transducer, as illustrated in Figure 2(c)), a parametric deformable model is employed to detect the luminal wall after spatial regularization (i.e. removal of small objects).

\section{EXPERIMENTAL RESULTS AND QUANTIFICATION}

In the first experiment, we studied the feasibility of our proposed technique by acquiring IVUS frames from a phantom cylinder using circulating flowing human blood. Figure 2 shows a selected constructed magnitude-phase histogram, binary masks for the two classes, and finally the automated detected phantom wall border. Secondly, we evaluated the algorithm performance on 205 IVUS frames acquired from two patients during catheterization procedure. Figure 3, illustrates a constructed magnitude-phase histogram for a single IVUS frame and automated detected luminal border along with manual traced contour by an expert. While the peaks are not as well separated as in the case of the phantom data they still provide good estimate of the relative magnitudes and phases for blood and non-blood regions and hence the detection of the lumen border in vivo. We quantified the results comparing the automated detected borders with manually traced contours by an expert. Statistics including true positive (TP), false positive (FP), and root mean square error (RMSE) rates are reported in Table II.

Table II. Quantification of automated detected lumen borders compared with corresponding expert manual tracings.

\begin{tabular}{|c|c|c|c|}
\hline Case \# & TP & FP & RMSE \\
\hline 1 & $88 \%$ & $4 \%$ & 20 \\
\hline 2 & $86 \%$ & $1 \%$ & 26.42 \\
\hline
\end{tabular}

\section{SUMMARY AND CONCLUSION}

In this paper, we presented a 3D segmentation framework for automatic detection of luminal borders in IVUS grayscale images by constructing the relative magnitudephase histogram of complex brushlet coefficients. These preliminary results demonstrated that our algorithm performed well, compared to manually traced borders by an expert. We plan to improve the spatial regularization part in the future and extend the approach to full vessel segmentation with different tissue layers.

\section{REFERENCES}

[1] M. Sonka, X. Zhang, M. Siebes, M. S. Bissing, S. C. DeJong, S. M. Collins, C. R. McKay, "Segmentation of Intravascular Ultrasound Images: A Knowledge-Based Approach,” IEEE Trans. Med. Imag. Vol. 14, No. 4, pp. 719-732, 1995.

[2] R. Shekhar, R. M. Cothren, D. G. Vince, S. Chandra, J. D. Thomas, J. F. Cornhill, "Three-Dimensional Segmentation of Luminal and Adventitial Borders is Serial Intravascular Ultrasound Images," Computerized Med. Imag. and Graphics, vol. 23, pp. 299-309, 1999.

[3] M. Plissiti, D. Fotidadis, L. Michalis, G. Bozios, "An Automated Methos for Lumen and Media-Adventitia Border Detection in a sequence of IVUS frames,” IEEE Trans. Info. Tech. Biomed., Vol. 19, No. 10, pp. 996-1011, 2000 .

[4] M. R. Cardinal, J. Meunier, G. Soulez, R. L. Maurice, E. Therasse, G. Cloutier, "Intravascular Ultrasound Image Segmentation: A ThreeDimensional Fast-Marching Method Based on Gray Level Distribution," IEEE. Trans. Med. Imag., Vol. 25, No. 5, pp. 590-601, 2006.

[5] G. Unal, S. Bucher, S. Carlier, G. Slabaugh, T. Fang, K. Tanaka, "ShapeDriven Segmentation of the Arterial Wall in Intravascular Ultrasound Images," IEEE Trans. Info. Tech. Biomed., Vol. 12, No. 3, pp. 335-347, 2008 .

[6] K. Hibi, A. Takagi, X. Zhang, T. T. Teo, H. N. Boneau, P. G. Yock, P. J. Fitzgerald, "Feasibility of a Novel Blood Reduction Algorithm to Enhance Reproducibility of Ultra-High-Frequency Intravascular Ultrasound Images," Circulation, Vol. 102, No. 14, pp. 1657-1663, 2000.

[7] S. Balocco, O. Basset, C. Cachard, P. Delachartre, "Spatial Aniosotropic Diffusion and Local Time Correlation Applied to Segmentation of Vessels in Ultrasound Image Sequences," IEEE Symp. Ultrasonics, Vol. 2, pp. 1549-1552, 2003.

[8] D. Rotger, P. Radeva, E. F. Nofrerias, J. Mauri, "Blood Detection in IVUS Images for 3D Volume of Lumen Changes Measurement Due to Different Drugs Administration," CAIP 2007, LNCS 4673, pp. 285-292.

[9] S. M. O’Malley, M. Naghavi, I. A. Kakadiaris, "One-Class Acoustic Characterization Applied to Blood Detection in IVUS," MICCAI 2007, Part I, LNCS 4791, pp. 202-209.

[10] A. Katouzian, E. Angelini, A. Lorsakul, B. Sturm, A. Laine, "Lumen Border Detection of Intravascular Ultrasound via Denoising of Directional Wavelet Representations," FIMH 2009.

[11] A. Katouzian, M. A. Selver, E. Angelini, B. Sturm, A. Laine, "Classification of Blood Regions in IVUS Images Using Three Dimensional Brushlet-Derived Features,” EMBC 2009.

[12] E. Angelini, A. Laine, S. Takuma, J. Holmes, S. Homma, "LV Volume Quantification via Spatio-Temporal Analysis of Real-Time 3D Echocardiography," IEEE Trans. Med. Imag., Vol. 20, No. 6, pp. 457-469, 2001.

[13] F. Meyer and R. R. Coifman, "Brushlets: A tool for directional image analysis and image compression," Applied and computational harmonic analysis, vol. 4, pp. 147-187, 1997.

[14] P. Ausher, G. Weiss, M. V. Wickerhauser, "Local sine and cosine bases of Coifman and Meyer and the construction of smooth wavelets," in Wavelets- A tutorial in Theory and Application, vol. 2, Wavelet Analysis and its Applications, C. K. Chui, Ed. San Diego: Academic Press, 1992, pp. 237-256. 